\title{
Prevalence of temporomandibular disorders in postmenopausal women and relationship with pain and HRT
}

\section{Victor Ricardo Manuel Muñoz LORA ${ }^{(\mathrm{a})}$ \\ Giancarlo De la Torre CANALES(a) Leticia Machado GONCCALVES(b) Carolina Beraldo MELOTO(c) Celia Marisa Rizzatti BARBOSA(a)}

(a) Universidade Estadual de Campinas - UNICAMP, Piracicaba Dental School, Department of Prosthesis and Periodontology, Piracicaba, São Paulo, Brazil.

(b)CEUMA University, Department of Prosthodontics, São Luis, Maranhão, Brazil.

(c) McGill University, The Alan Edwards Centre for Research on Pain, Montreal, Quebec, Canada.

Declaration of Interests: The authors certify that they have no commercial or associative interest that represents a conflict of interest in connection with the manuscript.

\section{Corresponding Author:}

Victor Ricardo Manuel Muñoz Lora E-mail: victor9874@hotmail.com

DOI: 10.1590/1807-3107BOR-2016.vol30.0100

Submitted: Aug 21, 2015

Accepted for publication: May 31, 2016

Last revision: June 26, 2016

\begin{abstract}
The prevalence of temporomandibular disorders (TMD) is higher in females, reaching their high peak during reproductive years, probably because of the action of some female hormones, which alter pain threshold. This study aimed to investigate the prevalence of TMD in postmenopausal women and its relationship with pain and hormone replacement therapy (HRT). In total, 284 patients were evaluated and classified using the Research Diagnostic Criteria for Temporomandibular Disorders (RDC/TMD). Pain was measured using the Visual Analogue Scale (VAS), and patients were also asked about the use of HRT. All data was analyzed using analysis of variance (ANOVA) and chi-square test. In total, 155 subjects did not have TMD and 129 had TMD; TMD group patients were classified according to RDC/TMD axis I classification as follows: muscle disorder group (1.6\%), disk displacement group (72.87\%), and arthralgia, osteoarthritis, and osteoarthrosis group (37.98\%). Pain was registered in 35 patients who belonged to the TMD group, while 48 patients reported the use of HRT. There was a similar percentage of TMD and non TMD patients; moreover, the use of exogenous hormones was no associated with TMD, suggesting that there is no influence on the pain threshold.
\end{abstract}

Keywords: Temporomandibular Joint Disorders; Epidemiology; Postmenopausa; Facial Pain; Estrogen Replacement Therapy.

\section{Introduction}

Temporomandibular disorders (TMD) comprise a set of clinical conditions related to the temporomandibular joints (TMJ) and masticatory muscles of the face and neck. These conditions are frequently related to other structures of the body, complicating their classification, diagnosis, and treatment $t^{1,2,3}$.

The prevalence of TMD symptoms ranges from $16 \%$ to $59 \%$ in studies of non-patient populations ${ }^{4,5}$. On the other hand, clinical studies have reported higher prevalences ( $45 \%$ to $50 \%$ ) and are more likely to identify severely affected patients ${ }^{6,7,8}$. Cross-sectional epidemiological studies of specific populations have shown that about $75 \%$ have at least one sign of TMD and about $33 \%$ have at least one symptom ${ }^{9,10}$, with pain being the predominant symptom. 
Approximately $7 \%-15 \%$ of the adult population in North America is afflicted with TMD ${ }^{11,12}$, and it is believed that approximately 6 million Brazilians have signs and symptoms of these disorders. Most, however, are not aware of the disorder that may be affecting them or of any sort of possible treatment or prognosis ${ }^{13}$.

Although the epidemiology of TMD has been studied $^{6}$, reported prevalence rates vary broadly, reflecting important differences in samples, criteria, and methods used for collecting the information ${ }^{14,15}$.

Epidemiological studies suggest that symptoms related to TMD predominantly occur in young adults and in females ${ }^{14,16}$. Studies have reported a female:male ratio of approximately $4: 1$ in terms of the prevalence of TMD ${ }^{10}$. Furthermore, this condition has the highest prevalence among women in their reproductive years (aged 20 to 40 years) ${ }^{17}$ and lowest prevalence among postmenopausal women, children, adolescents, and the elderly ${ }^{18,19}$. This higher prevalence among women has been attributed to inflammatory responses to stress, sociocultural behavior in response to pain, and effects related to hormonal characteristics ${ }^{14,16}$.

Several aspects of the prevalence pattern of TMD suggest that endogenous reproductive hormones play a role in these pain conditions, and various mechanisms explaining the influence of hormones in TMD pain can be postulated ${ }^{18}$. However, the exact mechanism of action of these hormonal effects remains unknown and is a matter of debate ${ }^{20,21}$.

The use of exogenous hormones and risk of TMD among postmenopausal women have been examined previously ${ }^{22}$. An examination of the records of a large health-maintenance organization revealed that the use of exogenous estrogen significantly increased the odds of having TMD. This study also reported that the probability of being a TMD case was approximately $30 \%$ higher among women receiving estrogen than among those not receiving estrogen and concluded that the use of oral contraceptives is associated with an approximately $20 \%$ increased risk of $\mathrm{TMD}^{15}$.

Accordingly, the aim of this study was to assess and classify the prevalence of TMD in postmenopausal women and the relationship(s) between hormone replacement therapy (HRT), TMD, and pain.

\section{Methodology}

This study used data collected from 284 postmenopausal women who were seeking different treatments at the Post-Graduation Clinic, Piracicaba Dental School, University of Campinas, between October 2013 and February 2015. The study was approved by the Ethics Committee of the Piracicaba Dental School, and all patients gave their informed consent to participate.

The sample-size requirement was calculated using standard formulas for finite population sampling, considering $\alpha=.05, \beta=.20$, and a sampling error of $10 \%$. The minimum sample size was estimated at 180 on the basis of data presented by Nekora-Azak et al. ${ }^{23}$

Four trained examiners were responsible for obtaining patient history and conducting the clinical examination, according to the RDC/TMD guidelines available on the RDC-TMD consortium website ${ }^{24}$ since 2002. Patients who did not have a menstrual period for at least 12 consecutive months were included in the study; exclusion criteria were polyarthritis or other rheumatic disease or any other orofacial pain disorder.

For this study, only axis I of the RDC/TMD was considered, including three different diagnosis groups: muscle disorders (group I), disk displacement (group II), and arthralgia, osteoarthritis, and osteoarthrosis (group III). Patients were then classified as belonging to none, one, or more than one group, depending on the signs and symptoms identified through the historytaking and clinical analysis conducted by more than one examiner at the same time.

After classification according to these RDC/TMD groups, patients were asked about the use of HRT using a question with a yes/no response, and the presence of pain was assessed in all patients using the Visual Analogue Scale (VAS). For VAS, each patient was presented with a paper on which a $10-\mathrm{cm}$-long line was drawn, marked at one end with 0 and at the other end with 10; the patients were asked to point to the position on the line that indicated the degree of pain they were feeling at the moment, considering 0 as the absence of pain and 10 as the highest pain they could feel. The distance between 0 and the point indicated was measured and recorded. 
Analysis of variance (ANOVA) was used to analyze the frequency and mean age of patients with single and combined RDC/TMD axis I diagnoses. The correlation between the use of HRT and signs of pain was assessed using a chi-square test.

\section{Results}

Classifying the patients according to RDC/TMD yielded 155 subjects (54.58\%) without TMD and 129 subjects $(45.42 \%)$ with TMD; their mean age was 56.7 years. Moreover, VAS testing identified 249 patients $(87.68 \%)$ with no pain symptoms and 35 patients (12.32\%) with moderate or severe pain symptoms. Among all these postmenopausal women, 204 (71.83\%) were not undergoing HRT and 80 (28.17\%) were undergoing HRT (Figure 1).

Patients identified without TMD were also without pain. Among this TMD-negative group (total, 155), 42 (27.10\%) were undergoing HRT, whereas 113 (72.90\%) were not undergoing any hormone treatment (Figure 1). In contrast, 94 (72.87\%) subjects identified as having TMD (total 129) reported no or low pain, whereas 35 $(27.13 \%)$ reported moderate to severe pain. In addition, 46 (35.66\%) were undergoing HRT and 83 (64.34\%) were not receiving hormone treatment. From the TMD + pain group ( 35 subjects), 57.14\% subjects were undergoing HRT, whereas $42.86 \%$ were not (Figure 1). The chisquare test $(p<0.05)$ showed no significant differences between the use of HRT and signs of pain (Table 1).

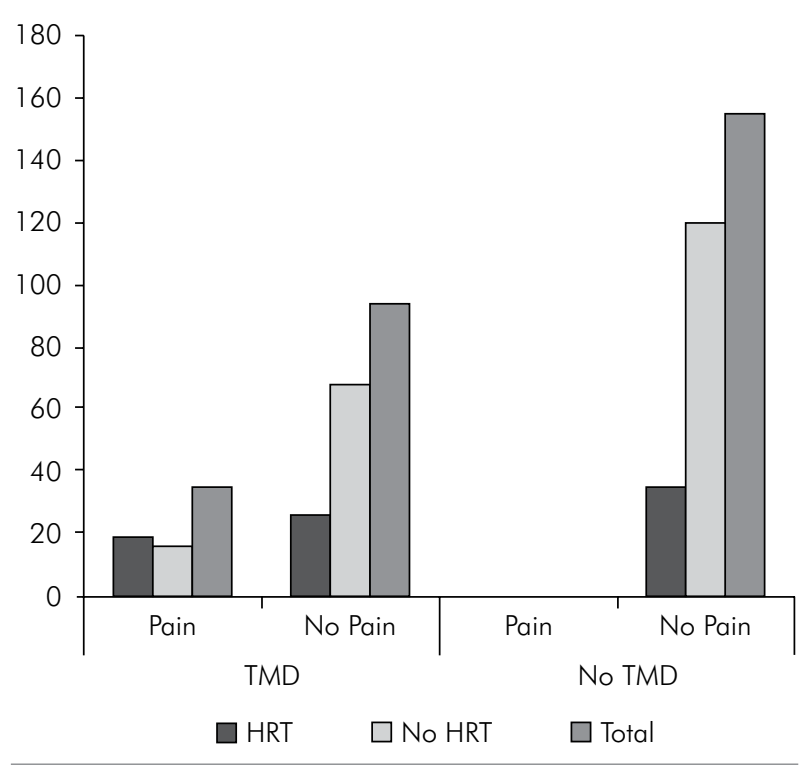

Figure 1. TMD, pain, and HRT relationship.
On classifying the TMD-positive patients into the three RCD/TMD-defined TMD groups (one patient fell into more than one group), two patients (1.6\%) were assigned to group I (muscle disorders), both (100\%) of which reported pain symptoms; 94 patients $(72.87 \%)$ were assigned to group II (disk displacement), 12.77\% (12 patients) of which reported pain symptoms, while $87.23 \%$ (82 patients) did not; and 49 patients (37.98\%) were assigned to group III (arthralgia, osteoarthritis, and osteoarthrosis), $71.23 \%$ (35 patients) of which reported pain symptoms, while $28.57 \%$ (14 patients) did not (Figure 2).

The frequency and mean age of single and combined RDC/TMD diagnoses were calculated using ANOVA $(p<0.05)$; the results are shown in Table 2. Group I was not diagnosed alone; the disk displacement group (group II) consisted of 28 patients, $17 \%$ of which had a mean age of $57.71 \pm 8.42$ years, while group III consisted of 12 patients, $68 \%$ of which had a mean age of $58.28 \pm 7.73$ years. Three patients had combined diagnoses of disk displacements (group II) and arthralgia, osteoarthritis, and osteoarthrosis (group III); $87 \%$ of these had a mean age of $52.18 \pm 6.95$ years. Patients diagnosed in all groups together were present in $0,7 \%$ of cases, with a mean age of $53.00 \pm 2.83$ years. No other diagnostic combinations were present in the study.

Table 1. Correlation between TRH use and signs of pain.

\begin{tabular}{lcccc}
\hline TRH & $\begin{array}{c}\text { Without } \\
\text { TMD }\end{array}$ & $\begin{array}{c}\text { TMD } \\
\text { without pain }\end{array}$ & $\begin{array}{c}\text { TMD } \\
\text { with pain }\end{array}$ & p-value \\
\hline No & $113(72.90 \%)$ & $68(72.34 \%)$ & $15(42.86 \%)$ & 0.1841 \\
Yes & $42(27.10 \%)$ & $26(27.66 \%)$ & $20(57.14 \%)$ & \\
\hline \multicolumn{4}{l}{ Chi-square test at a significance level set at $5 \%}$.
\end{tabular}

Table 2. Frequency and mean age of single and combined RDC/TMD axis I diagnoses in the study population.

\begin{tabular}{lcc}
\hline $\begin{array}{l}\text { RDC/TMD axis I group } \\
\text { diagnoses }\end{array}$ & \% Frequency & Mean age* \\
\hline No TMD & 54.58 & $56.81 \pm 9.33$ \\
I & 0.0 & - \\
II & 28.17 & $57.71 \pm 8.42$ \\
III & 12.68 & $58.28 \pm 7.73$ \\
I + II & 0.0 & - \\
I + III & 0.0 & - \\
II + III & 3.87 & $52.18 \pm 6.95$ \\
I + II + III & 0.70 & $53.00 \pm 2.83$ \\
\hline
\end{tabular}

*There were not significant differences in patients with single and combined diagnoses. 


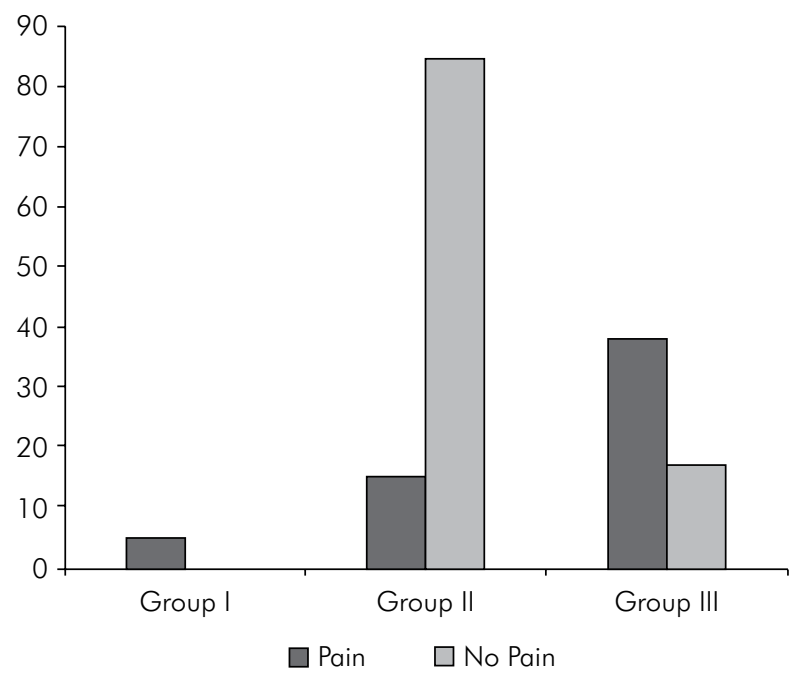

Figure 2. RDC/TMD division groups and pain relationship.

\section{Discussion}

It is well known that the prevalence of TMD is higher in females than in males ${ }^{15,25,26}$; this is probably due to the presence of certain gender hormones, which decrease after menopause, reducing TMD incidence $^{27,28}$. There is a concern about the absence of TMD epidemiology involving postmenopausal women; this issue complicates the comparison of findings from different studies.

RDC/TMD is a standardized diagnostic guideline that helps in the assessment of patients with TMD ${ }^{29}$ and has been the most widely employed protocol since $1992^{30}$. A new version, now called DC/TMD, has been released, but it was not available when this study was conducted. Although this new protocol provides some advantages over the old one, especially facilitating the diagnostics by clinicians, RDC/TMD has proved its reliability with different studies, including a multicenter one conducted by John et al. ${ }^{31}$. These data, in addition to the fact that the present study was performed by capacitated and trained researchers, support the reliability of the reported results.

This study revealed a similar percentage of women with TMD (54.58\%) and without TMD (45.42\%). In addition, only $27.13 \%$ of patients from the TMD group experienced some pain during clinical examination.

Eighty-three patients from the TMD group (total, 129 subjects) and 113 patients from the no TMD group (total, 155 subjects) were not under HRT, suggesting no relationship between the use of HRT and the presence of TMD; this fact is in accordance with that revealed in study of Nekora-Azak et al. ${ }^{23}$; that study revealed no differences in the relationship between postmenopausal hormone use and TMD in 180 postmenopausal women. Conversely, a study conducted by LeResche et al. ${ }^{17}$ contradicted this finding, indicating a strong evidence for a relationship between hormone use and TMD; it also mentioned that the relationships between hormone use and TMD would not be observed in a random sample of TMD cases identified in the community, which would be a limitation of the study.

LeResche et al. ${ }^{17}$ have also indicated the existence of an increased risk of TMD pain among women using various forms of exogenous hormones; this fact is contradictory to the findings of our study, where there was a similar percentage of HRT patients $(57,14 \%)$ and no HRT patients $(42,86 \%)$ in the TMD + pain group ( 35 subjects), suggesting no relationship between the use of exogenous hormones and TMD pain. It is probably due to the sample size and because those authors collected data from record forms, instead of performing clinical evaluations, as conducted in our study.

When classifying TMD patients (129 subjects) into RDC/TMD groups, most patients (72.87\%) were classified into group II (disk displacement); probably this fact is because of an increase in intra-articular ligament laxity, which is related to aging; the minority of them $(1.6 \%)$ were classified into group I (muscle disorders). In contrast, a study conducted with 462 DTM patients by Manfredini et al. ${ }^{29}$ classified the majority of patients (57.5\%) into group III and the minority of them (42\%) into group II; the difference between these studies may be due to the age range of patient selection and the TMD sample size, which was one of the limitations of this study.

The authors consider that the sample size may have influenced in the results. Similarly, measuring hormone quantity by specific blood tests could provide more reliable results.

Further, others studies should be developed considering the application of RDC/TMD axis II to investigate the influence of behavioral and psychological factors on increase in pain ${ }^{22}$. The authors believe that more epidemiological studies should be conducted to confirm the results of the present study. 


\section{Conclussion}

In summary, the group II (disk displacement) from the RDC/TMD axis I was diagnosed more frequently than the other two groups, indicating that disk displacement disorders tend to be more common with aging. In addition, TMD and the

\section{References}

1. Oliveira W. Disfunção temporomandibular. São Paulo: Artes Médicas; 2002.

2. Di Paolo C, Costanzo GD, Panti F, Rampello A, Falisi G, Pilloni A et al. Epidemiological analysis on 2375 patients with TMJ disorders: basic statistical aspects. Ann Stomatol (Roma). 2013;4(1):161-9. doi:10.11138/ads.0161

3. Rammelsberg P, LeResche L, Dworkin S, Mancl L. Longitudinal outcome of temporomandibular disorders: a 5-year epidemiologic study of muscle disorders defined by research diagnostic criteria for temporomandibular disorders. J Orofac Pain. 2003;17(1):9-20.

4. Schiffman EL, Fricton JR, Haley DP, Shapiro BL. The prevalence and treatment needs of subjects with temporomandibular disorders. J Am Dent Assoc. 1990;120(3):295-303. doi:10.14219/jada.archive.1990.0059

5. Carlsson GE. Epidemiology and treatment need for temporomandibular disorders. J Orofac Pain. 1999;13(4):232-7.

6. Bevilacqua-Grossi D, Chaves TC, Oliveira AS, Monteiro- Pedro V. Anamnestic index severity and signs and symptoms of TMD. Cranio. 2006;24(2):112-8. doi:10.1179/crn.2006.018

7. Casanova-Rosado JF, Medina-Solís CE, VallejosSánchez AA, Casanova-Rosado AJ, Hernández-Prado B, Avila-Burgos L. Prevalence and associated factors for temporo- mandibular disorders in a group of Mexican adolescents and youth adults. Clin Oral Invest. 2006;10(1):42-9. doi:10.1007/s00784-005-0021-4

8. Bonjardim LR, Lopes-Filho RJ, Amado G, Albuquerque RL Jr, Gonçalves SRJ. Association between symptoms of temporomandibular disorders and gender, morphological occlusion, and psychological factors in a group of university students. Indian J Dent Res. 2009;20(2):190-4. doi:10.4103/0970-9290.52901

9. Okeson JP, editor. Orofacial pain: guidelines for assessment, diagnosis, and management. Chicago: Quintessence; 1996.

10. Atsü SS, Ayhan-Ardic F. Temporomandibular disorders seen in rheumatology practices: a review. Rheumatol Int. 2006;26(9):781-7. doi:10.1007/s00296-006-0110-y

11. Lipton JA, Ship JA, Larach-Robinson D. Estimated prevalence and distribution of reported orofacial pain in the United States. J Am Dent Assoc. 1993;124(10):115-21. doi:10.14219/jada.archive.1993.0200 pain caused by this presence seem to have no relationship with the use of HRT, as suggested in some previous studies. More epidemiological studies should be developed, and the use of RDC/TMD axis II to evaluate behavioral and psychological factors is recommended.

12. Goulet JP, Lavigne GJ, Lund JP. Jaw pain prevalence among French-speaking Canadians in Québec and related symptoms of temporomandibular disorders. J Dent Res. 1995;74(11):1738-44. doi:10.1177/00220345950740110401

13. Shibayama R, Garcia AR, Zuim PR. [Prevalence of temporomandibular disorder symptons in complete dentures, removable partial denture patients and university students]. Rev Odontol Araçatuva. 2004;25(2):18-21. Portuguese.

14. Gonçalves DA, Dal Fabbro AL, Campos JA, Bigal ME, Speciali JG. Symptoms of temporomandibular disorders in the population: an epidemiological study. J Orofac Pain. 2010;24(3):270-8.

15. LeResche L. Epidemiology of temporomandibular disorders: implications for the investigation of etiologic factors. Crit Rev Oral Biol Med. 1997;8(3):291-305. doi:10.1177/10454411970080030401

16. Campos JA, Carrascosa AC, Bonafé FS, Maroco J. Epidemiology of severity of temporomandibular disorders in Brazilian women. J Oral Facial Pain Headache. 2014;28(2):147-52. doi:10.11607/ofph.1194

17. Von Korff M, Dworkin SF, Le Resche L, Kruger A. An epidemiologic comparison of pain complaints. Pain. 1988;32(2):173-83. doi:10.1016/0304-3959(88)90066-8

18. L. LeResche L, Saunders K, Von Korff MR, Barlow W, Dworkin SF. Use of exogenous hormones and risk of temporomandibular disorder pain. Pain. 1997;69(1-2):15360. doi:10.1016/S0304-3959(96)03230-7

19. Kuttila M, Niemi PM, Kuttila S, Alanen P, Le Bell Y. TMD treatment need in relation to age, gender, stress, and diagnostic subgroup. J Orofac Pain. 1998;12(1):67-74.

20. Ribeiro-Dasilva MC, Line SRP, Santos MCLG, Arthuri MT, Hou W, Fillingim RB et al. Estrogen receptor-alpha polymorphisms and predisposition to TMJ disorder. J Pain. 2009;10(5):527-33. doi:10.1016/j.jpain.2008.11.012

21. Guan G, Kerins CC, Bellinger LL, Kramer PR Estrogenic effect on swelling and monocytic receptor expression in an arthritic temporomandibular joint model. J Steroid Biochem Mol Biol. 2005;97(3):241-50. doi:10.1016/j.jsbmb.2005.05.013

22. Warren, M.; Fried, J. Temporomandibular disorders and hormones in women. Cells Tissues Organs. 2001;169(3):187-92. doi:10.1159/000047881

23. Nekora-Azak A, Evlioglu G, Ceyhan A, Keskin $\mathrm{H}$, Berkman S, Issever $\mathrm{H}$. Estrogen replacement therapy among postmenopausal women and its effects on signs and symptoms of temporomandibular disorders. Cranio. 2008;26(3):211-5. doi:10.1179/crn.2008.028 
24. International RDC-TMD Consortium. TMD assessment/diagnosis. 2014 [access 2014 June 15]. Available from: http://www.rdctmdinternational.org/ TMDAssessmentDiagnosis/RDCTMD/Translations/Italian.aspx.

25. Locker D, Slade G. Prevalence of symptoms associated with temporomandibular disorders in a Canadian population. Community Dent Oral Epidemiol. 1988;16(5):310-3. doi:10.1111/j.1600-0528.1988.tb01783.x

26. Dworkin SF, Huggins KH, LeResche L, Von Korff M, Howard J, Truelove E et al. Epidemiology of signs and symptoms in temporomandibular disorders: clinical signs in cases and controls. J Am Dent Assoc. 1990;120(3):273-81. doi: 10.14219/jada.archive.1990.0043

27. Ganshorn ML, Gärtner F. [Studies on possible correlations between diseases of temporomandibular joint and female sex hormones[. ZWR. 1975;84(16):726-8. German.
28. Licini F, Nojelli A, Segù M, Collesano V. Role of psychosocial factors in the etiology of temporomandibular disorders: relevance of a biaxial diagnosis. Minerva Stomatol. 2009;58(11-12):557-66.

29. Manfredini D, Arveda N, Guarda-Nardini L, Segù M, Collesano V. Distribution of diagnoses in a population of patients with temporomandibular disorders. Oral Surg Oral Med Oral Pathol Oral Radiol. 2012;114(5):e35-41. doi:10.1016/j.oooo.2012.03.023

30. Dworkin SF, LeResche L. Research diagnostic criteria for temporomandibular disorders: review criteria, examinations and specifications, critique. J Craniomandib Disord. 1992;6(4):301-55.

31. John MT, Dworkin SF, Mancl LA. Reliability of clinical temporomandibular disorder diagnoses. Pain. 2005;118(1-2):61-9. doi:10.1016/j.pain.2005.07.018 